\title{
Verticillin A suppresses HGF-induced migration and invasion via repression of the c-Met/FAK/Src pathway in human gastric and cervical cancer cells
}

This article was published in the following Dove Press journal: OncoTargets and Therapy

\author{
Jingxin $\mathrm{Lu}^{\mathrm{I}, 2, *}$ \\ Xia $\mathrm{Li}^{2,3, *}$ \\ Kai Tu ${ }^{1,2}$ \\ Yuelin Guan ${ }^{1,2}$ \\ Kwok-Pui Fung ${ }^{2,4}$ \\ Feiyan Liu ${ }^{\text {I,2 }}$ \\ 'Research Centre of Siyuan Natural \\ Pharmacy and Biotoxicology, College of \\ Life Sciences, Zhejiang University, \\ Hangzhou 310058, People's Republic of \\ China; ${ }^{2}$ Joint Centre of Zhejiang \\ University and the Chinese University of \\ Hong Kong on Natural Products and \\ Toxicology Research, Zhejiang University, \\ Hangzhou, People's Republic of China; \\ ${ }^{3}$ Zhejiang Cancer Research Institute, \\ Zhejiang Cancer Hospital, Hangzhou \\ 310022, People's Republic of China; \\ ${ }^{4}$ School of Biomedical Sciences (SBS), \\ The Chinese University of Hong Kong, \\ Shatin, Hong Kong SAR, People's \\ Republic of China
}

*These authors contributed equally to this work
Background and purpose: Verticillin A is a fungal epipolythiodioxopiperazine (ETP) metabolite that was isolated from Amanita flavorubescens Alk infected by Verticillium sp. It was previously proven to possess potent anti-tumor cell growth activity, and we have recently determined that verticillin $\mathrm{A}$ is a selective inhibitor of $\mathrm{H} 3 \mathrm{~K} 9 \mathrm{me} 3$-specific histone methyltransferase. The objective of this study was to find out whether verticillin $\mathrm{A}$ is an effective agent for suppression of gastric and cervical tumor progression.

Materials and methods: Wound healing and transwell assays was performed to evaluate the effect of verticillin A on hepatocyte growth factor (HGF)-induced AGS and HeLa cells migration and invasion in vitro. Western blot was used to detect signaling proteins verticillin A affected.

Results: We determined that verticillin A effectively suppressed hepatocyte growth factor (HGF)induced AGS and HeLa cells migration and invasion in vitro. At the molecular level, we demonstrated that verticillin A inhibited HGF-induced c-Met phosphorylation and repressed the expression of total c-Met protein in AGS and HeLa cells, resulting from reduced expression of fatty acid synthase. In addition, verticillin A could suppress c-Met downstream FAK/Src signaling pathways by impairing c-Met phosphorylation induced by HGF.

Conclusion: Our study demonstrated verticillin A inhibits the migration ability of human gastric cancer (AGS) cells and cervical cancer (HeLa) cells by targeting c-Met and its downstream FAK/Src signaling pathways, and suggested that verticillin A acts as a novel $\mathrm{HGF} / \mathrm{c}-\mathrm{Met}$ inhibitor by reducing expression of this receptor.

Keywords: verticillin A, gastric and cervical cancers, cell migration, c-Met, FAK

\section{Introduction}

Cancer is a complex disorder, which has a high-mortality rate worldwide. Gastric cancer is one of the most common human malignant diseases, and men have much higher rates of gastric cancer incidence and mortality than women. There were $>70 \%$ deaths due to gastric cancer in 2012, making this malignancy the third leading cause of cancer-related mortality worldwide. ${ }^{1,2}$ Cervical cancer remains the second most common cancer and third leading cause of cancer death among females, and it is the most frequent in developing countries. ${ }^{2}$ Human papillomavirus (HPV) DNA can be detected in $95-100 \%$ of adequate specimens of cervical cancer, suggesting that the primary cause in the development of cervical cancer is HPV. ${ }^{3}$

Treatment of cancer mainly includes surgery, adjuvant chemotherapy, and radiation therapy. ${ }^{4}$ Targeted therapies show promising activity in cancer therapy in recent years. Although recent diagnostic and therapeutic advances have improved the clinical
Research Centre of Siyuan Natural

Pharmacy and Biotoxicology, College of Life Sciences, Zhejiang University, 866

Yu-hang-tang Road, Hangzhou 310058,

People's Republic of China

Tel +865718 8206287

Fax +865718 8206287

Email liuf64@zju.edu.cn 
outcomes of patients with early stage of cancer, metastatic dissemination is the most life-threatening aspect of cancer. ${ }^{5}$ It represents a major problem in the treatment of cancer and it still remains the most common cause of death in cancer patients. ${ }^{6}$

Metastasis is a very intricate process, involving cell adhesion, migration, and proliferation. Yet, it remains the most poorly understood component of cancer pathogenesis. However, there are some general principles in the metastatic cascade among the various cancer types. It has been reported that metastasis can be simplified into two major phases: 1) physical translocation of cancer cells to distant organs and 2) develop into a metastatic lesion at that distant sites. ${ }^{6,7}$

The role of hepatocyte growth factor (HGF)/c-Met pathway in metastasis has been gaining interest.

c-Met, the receptor tyrosine kinase encoded by $M E T$ proto-oncogene located on chromosome 7q21-q31, is expressed in epithelial cells of many organs. The only known ligand for c-Met is HGF. ${ }^{8}$ Upon binding by HGF, c-Met becomes phosphorylated and acts as the multifunction docking site for recruiting intracellular effector proteins, propagating a signaling cascade, which include the ERK/MAPK, PI3K/Akt, PIP3-PKC, STAT3, FAK and JNK pathways. ${ }^{9}$ Particularly, FAK has been reported to act as a key signal mediator in tumor progression and metastasis. ${ }^{10}$ Currently, various studies have shown that the HGF/c-Met pathway is frequently dysregulated in many types of cancer. ${ }^{11-13}$ Interestingly, activation, overexpression, or genetic aberrations of c-Met are associated with the proliferation, invasion, angiogenesis as well as protection from apoptosis in cancer cells. Therefore, c-Met has been recognized as a therapeutic target for targeting tumor metastasis. ${ }^{14}$ Several compounds have been developed to target the HGF/c-Met pathway, including antibodies against HGF, MET receptors, and small molecule MET inhibitors. ${ }^{15,16}$

Verticillin A, a natural compound purified from Verticillium-infected mushrooms Amanita flavorubescens Alk, possesses potent anti-tumor cell growth activity in vitro and in vivo. ${ }^{17}$ Verticillin $\mathrm{A}$ is an $\mathrm{H} 3 \mathrm{~K} 4 \mathrm{me} 3-$ and H3K9me3-selective histone methyltransferase and functions as a potent apoptosis sensitizer to confer tumor cell sensitivity to cell death induction by TRAIL and 5fluorouracil. $^{17,18}$ In the present study, we demonstrated that verticillin $A$ inhibits the c-Met/FAK/Src signaling pathway through repression of c-Met expression to suppress gastric and cervical cancer migration and invasion. Our findings determined that verticillin $\mathrm{A}$ is a natural compound that holds great potential for further development for the treatment of metastasis of human cancers.

\section{Materials and methods}

\section{Cell lines}

The human gastric cancer cells AGS and cervical cancer cells HeLa were obtained from American Type Culture Collection (Manassas, VA, USA). The cells were cultured in high glucose DMEM (Gibco, Grand Island, NY, USA) supplemented with $10 \%$ FBS, penicillin-streptomycin (Gibco) in a humidified atmosphere of $5 \% \mathrm{CO}_{2}$ at $37^{\circ} \mathrm{C}$. The cells were routinely sub-cultured every 2 or 3 days, and all of the cell samples were verified to be in the logarithmic growth phase.

\section{Reagents}

Verticillin A was purified from wild mushroom with a purity of $>99 \%$ as previously described. ${ }^{17} \mathrm{C} 75$ and palmitate were purchased from Sigma-Aldrich (St Louis, MO, USA). HGF was purchased from Sino Biological (Beijing, China). The primary antibodies for c-Met, p-c-Met (Y1234/1235), FAK, p-FAK (Y925), Src and p-Src (Y416) were obtained from Cell Signaling Technology (Danvers MA, USA). The primary antibody for $\beta$-actin was obtained from SigmaAldrich, and HPR-conjugated secondary antibodies were obtained from Santa Cruz Biotechnology (Santa Cruz, CA, USA).

\section{Cell viability assay}

Cell viability and proliferation were determined by MTT assay. Cells were seeded in 96-well plate and allowed to adhere overnight. Then, cells cultured in the presence of various concentrations of verticillin A for 24 and $48 \mathrm{hrs}$, followed by addition of $50 \mu \mathrm{L}$ MTT $(1 \mathrm{mg} / \mathrm{mL})$ and incubation for another $4 \mathrm{hrs}$. The cells were then treated with 150 $\mu \mathrm{LDMSO}$ to dissolve the formazan crystals. The plates were then read in a microplate reader at $570 \mathrm{~nm}$.

\section{Wound-healing assay}

Cells were seeded in a 6-well plate to form a confluent monolayer overnight in serum-containing media. The cell monolayer was then gently and slowly scratched across the center of the well with a sterile $10 \mu \mathrm{L}$ pipette tip. After scratching, the detached cells were removed by washing with PBS gently for three times. Cells were then incubated with various concentrations of verticillin A. HGF $(40 \mathrm{ng} / \mathrm{mL})$ were added to the cell culture 24 and 48 hrs later, respectively, in serum-free medium. The 
recording sites of each well were marked, and photos were taken at the marked sites on a microscope at 0,24 and $48 \mathrm{hrs}$ after addition of HGF. The distance traveled by the cells was determined by measuring the wound width.

\section{Cell migration and invasion assays}

The cell migration and invasion capability were measured by using transwell chambers (Corning Incorporated, Corning, $\mathrm{NY}$, USA) with $8 \mu \mathrm{m}$ pore-size filters coated without or with matrigel basement membrane matrix. AGS and HeLa cells were suspended in serum-free DMEM medium and $100 \mu \mathrm{L}$ of the cells suspension was added to the top of the transwell inserts, and $500 \mu \mathrm{L}$ of serum-containing medium with or without the addition of HGF $(40 \mathrm{ng} / \mathrm{mL})$ was plated in the bottom wells. Verticillin A was added to both inserts and bottom wells. The chambers were then assembled and incubated at $37^{\circ} \mathrm{C}$ in a $5 \% \mathrm{CO}_{2}$ incubator. After $24 \mathrm{hrs}$, noninvading cells were removed from the upper surface of the membrane with a cotton swab. The migrated cells on the lower surface of the insert were first fixed with 100\% methanol and then stained by $0.1 \%$ crystal violet solution and counted in five random fields. The cell invasion assay is similar as described above with the inclusion of matrigel pre-coated transwell chambers. Briefly, Matrigel Basement Membrane Matrix (Corning, Bedford, MA, USA) was dissolved on ice and diluted with serum-free DMEM medium (1:40). After matrigel coating, the inserts were then incubated at $37^{\circ} \mathrm{C}$ for 30 mins. Cell invasion was measured as the cell migration as described.

\section{Western blotting analysis}

AGS and HeLa cells were incubated with verticillin A and/or HGF. Cells were lysed in lysis buffer $[2.1 \mu \mathrm{g} / \mathrm{mL}$ Aprotinin, $0.5 \mu \mathrm{g} / \mathrm{mL}$ Leupeptin, $4.9 \mathrm{mM} \mathrm{MgCl} 2,1 \mathrm{mM}$ orthovanadate, $1 \%$ Triton X-100, and $1 \mathrm{mM}$ phenylmethylsulfonyl fluoride]. The total lysate was centrifuged at $14,000 \mathrm{rpm}$ for $10 \mathrm{mins}$ at $4^{\circ} \mathrm{C}$. Protein concentration was determined by the BCA assay [Reagent A: Pierce BCA Protein Assay (Thermo Scientific, Rockford, IL, USA), Reagent B: $\left.4 \% \quad \mathrm{CuSO}_{4} \cdot 4 \cdot 5 \mathrm{H}_{2} \mathrm{O}\right]$. Equivalent amounts of protein were electrophoresed on 12\% SDSPAGE gels and transferred to PVDF membrane (Millipore, Billerica, MA, USA). The membranes were then blocked with $5 \%$ BSA, followed by incubation with a specific primary antibody overnight at $4^{\circ} \mathrm{C}$. Membranes were washed 3 times prior to incubate with secondary antibody.
Immune complexes were finally detected with enhanced chemiluminescence reagent (Pierce ECL Western Blotting Substrate; Thermo Scientific).

\section{FAK overexpression}

Plasmids pcDNA3.1(+)-FAK and negative control were chemically synthesized by Icartab (Jiangsu, China), pcDNA3.1 $(+)$-FAK and pcDNA3.1 were transfected using Lipofectamine 2000 (Invitrogen, Carlsbad, CA, USA) according to the manufacturer's instructions. Cells were transfected with plasmids for $24 \mathrm{hrs}$. After transfection, cells were treated with various concentrations of verticillin A for $24 \mathrm{hrs}$. Western blotting assay was then used to confirm the gene transfection efficiency. And, Transwell assay was performed to test cell migration ability transfected FAK.

\section{Statistical analysis}

Where indicated, data were represented as the mean $\pm \mathrm{SD}$ of at least three independent experiments. Statistical analysis was performed using two-sided $t$-test (two sample equal variance), with $P<0.05$ considered as statistically significant.

\section{Results}

Verticillin A suppresses human gastric and cervical cancer cell growth in vitro

Verticillin A inhibits histone methyltransferase SUV39H1 and MLL1 to decrease H3K4me3 and H3K9me3 depositions at a panel of apoptosis regulatory gene promoters to suppress pancreatic cancer cell growth in vitro. ${ }^{18,19}$ To determine whether verticillin A suppresses other cancer cell growth, human gastric (AGS) and cervical cancer cells (HeLa) were cultured in the presence of verticillin A at various concentrations for 24 and $48 \mathrm{hrs}$ in vitro. Analysis of cancer cell viability indicated that verticillin A inhibits AGS and HeLa cell growth in a dose- and time-dependent manner. Verticillin A was effective in AGS and HeLa cells with an $\mathrm{IC}_{50}$ of 69.89 and $319.5 \mathrm{nM}$, respectively, at $24 \mathrm{hrs}$. The $\mathrm{IC}_{50}$ values decreased to $47.59 \mathrm{nM}$ and $233.9 \mathrm{nM}$ for AGS and HeLa cells, respectively, at 48 hrs (Figure 1). We therefore conclude that verticillin $\mathrm{A}$ is a potent growth suppressor of human gastric and cervical cancer cells.

\section{Verticillin A treatment caused a dose- dependent suppression of HGF-stimulated cancer cell migration and invasion}

Apoptosis resistance confers tumor cells a survival advantage to progress. ${ }^{20}$ Furthermore, H3K4me3 and H3K9me3 
AGS

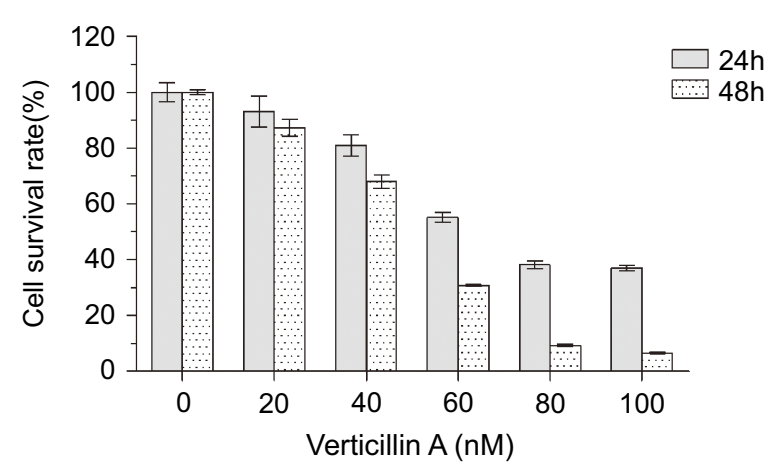

HeLa

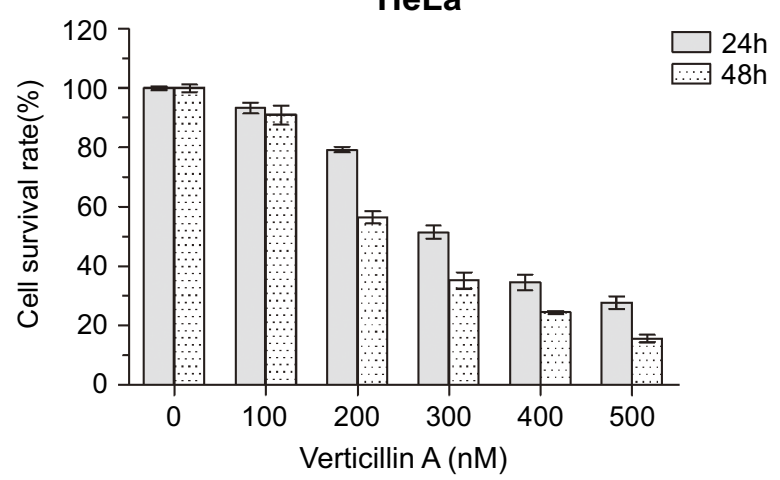

Figure I Verticillin A suppresses human gastric and cervical cancer cells growth in vitro. Human gastric AGS and Cervical HeLa cells were seeded in 96-well plates and cultured in the presence of various concentrations of verticillin A for 24 and 48 hrs, respectively. The cell viability was measured using the MTT assay. Data were mean \pm SD from three independent experiments with similar results.

both have been shown to promote tumor cell progression, ${ }^{21}$ we therefore sought to test the hypothesis that verticillin A is effective in suppression of tumor progression. To test this hypothesis, we used AGS and HeLa cells with HGF treatment to model tumor progression by measuring HGFinduced cancer cell migration and invasion in vitro. Verticillin A was added to the culture to determine its effects on cancer cell migration and invasion. Cell migration was determined by measuring wound closure and the migration rate. As expected, $\mathrm{HGF}$ at a concentration of 40 $\mathrm{ng} / \mathrm{mL}$ significantly induced the migration of both AGS (Figure 2A) and HeLa (Figure 2B) cells. A 48 hrs HGF treatment increased AGS and HeLa cellular migration rate by $54 \%$ and $42 \%$, respectively, as compared to untreated cells measured by wound-healing assay (Figure 2A and B). Verticillin A significantly inhibited this HGFinduced cellular migration in a dose-dependent manner (Figure 2A and B ). As shown in Figure 2A and B, in a $48 \mathrm{hrs}$ culture period, verticillin A decreased HGF-induced AGS cellular migration by $15 \%$ at a dose of $25 \mathrm{nM}$. Verticillin A treatment decreased HGF-induced AGS cellular migration by $32 \%$ at $50 \mathrm{nM}$. For HeLa cells, a higher dose is required to suppress cellular migration. Verticillin A at 100 and $200 \mathrm{nM}$ decreased HGF-induced HeLa cellular migration by $19 \%$ and $49 \%$, respectively, after $48 \mathrm{hrs}$ incubation.

The complimentary transwell assay was then used to validate the findings made with the wound-healing assay. Again, as expected, HGF-induced gastric and cervical tumor cells invasiveness as measured by transwell assay (Figure 2C and D) and verticillin A treatment significantly decreased this HGF-induced tumor cell invasiveness (Figure 2C and D). HGF induced a $>50 \%$ increase in both gastric and cervical tumor cells trans-membrane migration within 24 hrs. This HGF-induced tumor cells trans-membrane migration was significantly inhibited by verticillin A treatment in a dose-dependent manner (Figure 2C and D). We therefore determined that verticillin $\mathrm{A}$ is effective in suppression of tumor cells migration and invasion.

\section{Verticillin A blocks HGF-induced activation of the c-Met/FAK/Src signaling pathway}

It has been reported that aberrant activation of the HGF/c-Met signaling pathway is related to cellular migration and invasion to promote tumor cell metastasis. ${ }^{9,22}$ To determine whether verticillin A inhibits the c-Met signaling pathway to suppress cancer cell migration and invasion, we analyzed the effects of verticillin A on activation of c-Met protein in gastric and cervical cancer cells. AGS and HeLa cells were pretreated with verticillin A for $24 \mathrm{hrs}$ and then add HGF, after that the cell lysates were analyzed by Western blotting. Untreated AGS and HeLa cells have low level of c-Met phosphorylation (Tyr1234/1235), and HGF stimulation significantly increased c-Met phosphorylation. Verticillin A treatment significantly inhibited HGF-induced c-Met phosphorylation in a dosedependent manner (Figure 3A and B). Consistent with decreased c-Met phosphorylation, p-FAK (Tyr925) and p-Src (Tyr416), the downstream targets of c-Met were also significantly decreased in verticillin A-treated AGS and HeLa cells. The total FAK and Src protein levels were not changed significantly by verticillin A (Figure $3 \mathrm{C}$ and $\mathrm{D}$ ). Because the abnormal activation of the c-Met-FAK/Src signaling pathway is linked to cancer cell migration and invasion, ${ }^{23,24}$ our findings thus indicate that verticillin A inhibits the c-Met/FAK/Src signaling pathway activation to suppress human gastric and cervical cancer cells migration and invasion. 
A

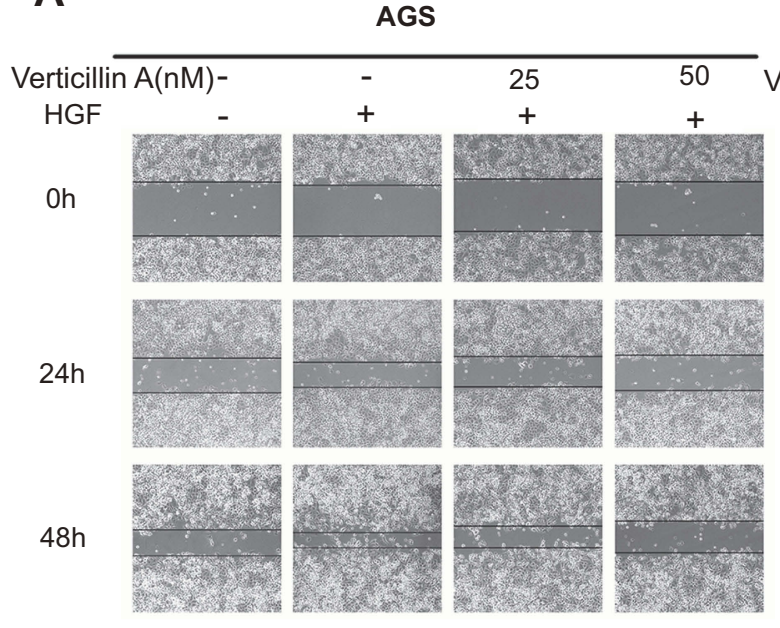

B

HeLa

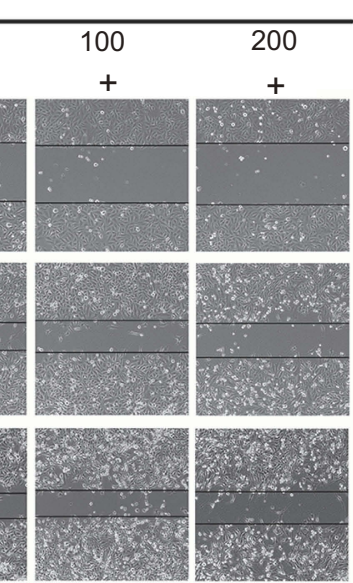

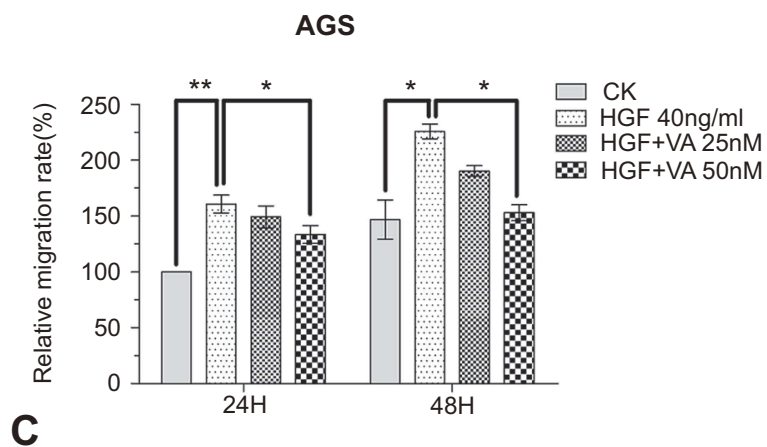
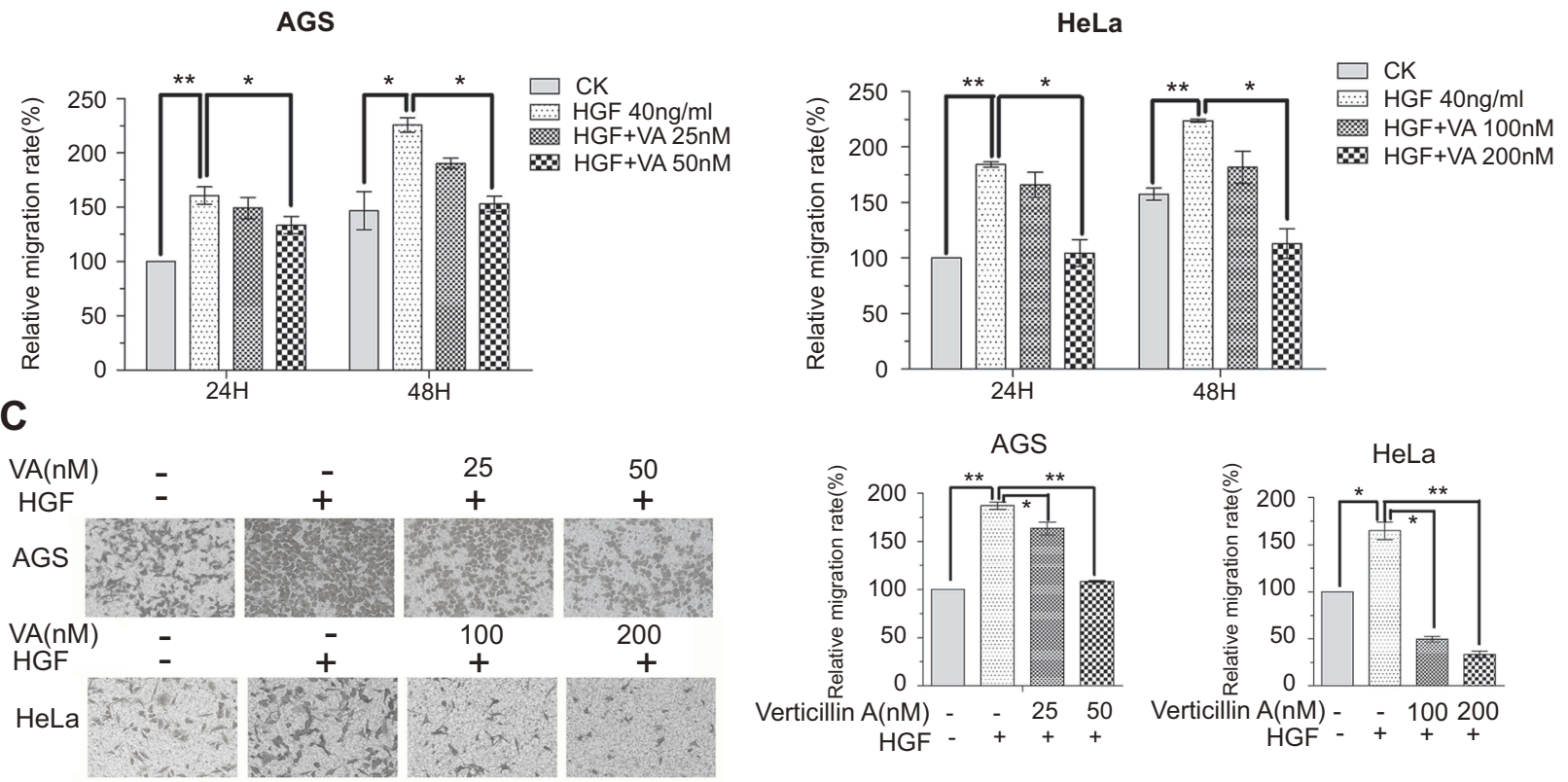

D
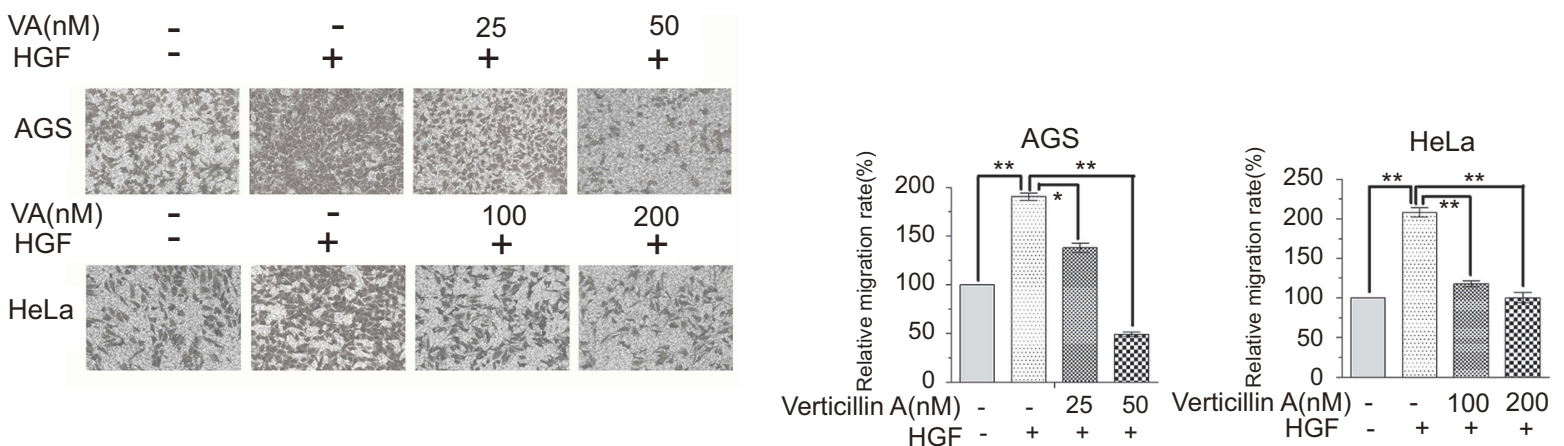

Figure 2 Verticillin inhibits HGF-induced migration and invasion of AGS and HeLa cells. (A \& B) AGS (A) and HeLa (B) cells were seeded in 6-well plates overnight to form monolayers, the monolayers were scratched by pipet tips and washed to remove the debris. Serum-free medium was added to the culture. Verticillin $A$ was then added at varying concentrations, and cells were cultured for 24 or $48 \mathrm{hrs}$, respectively, with (+) or without (-) $40 \mathrm{ng} / \mathrm{mL}$ HGF. Representative photomicrographs of wound-healing results were taken under $\times 100$ original magnification. (C \& D) AGS and HeLa cells were seeded onto the upper chamber consisting of $8 \mu \mathrm{m}$ pore-size filters coated without (C) or with (D) Matrigel basement membrane matrix, then treated with increasing doses of concentrations of verticillin A for 24 hrs with (+) or without $(-) 40$ ng/mL $\mathrm{HGF}$ in the lower chamber. The relative quantitative determinations of migrated and invasive cells were calculated with 5 fields counted per experiment taken under $\times 100$ original magnification factor. Data were shown as mean $\pm S D$ from three independent experiments. $* P<0.05, * * P<0.01$. VA represented Verticillin $A$ in this figure. 
A

\begin{tabular}{|c|c|c|}
\hline & AGS & HeLa \\
\hline Verticillin A(nM) & $-\quad-\quad 50100200$ & -100200400 \\
\hline HGF & -++++ & -++++ \\
\hline p-Met(Y1234/35) & 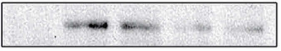 & $-\infty-m$ \\
\hline$\beta$-actin & $-m-\infty$ & $-\infty-\infty-1-\infty$ \\
\hline
\end{tabular}

B

AGS

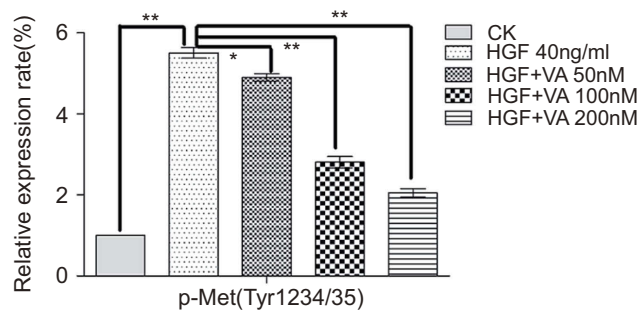

HeLa

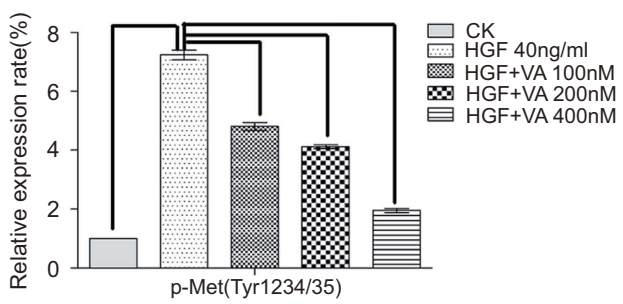

C
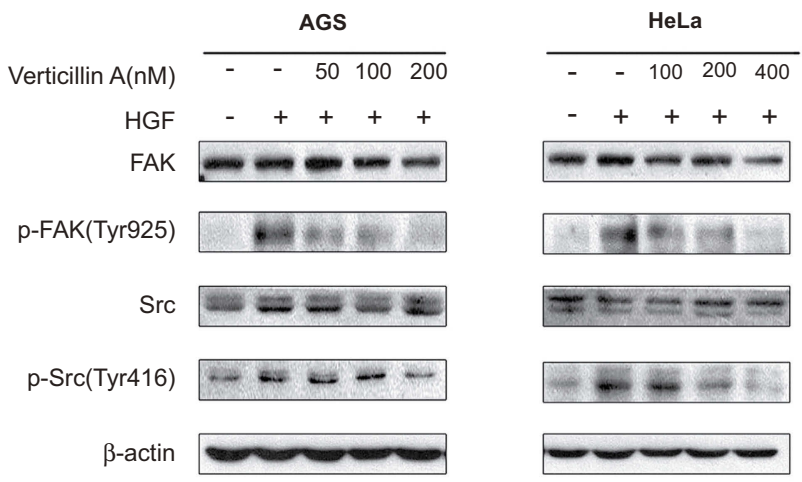

D

AGS

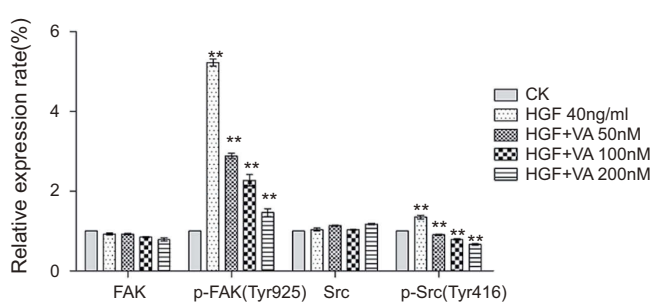

HeLa

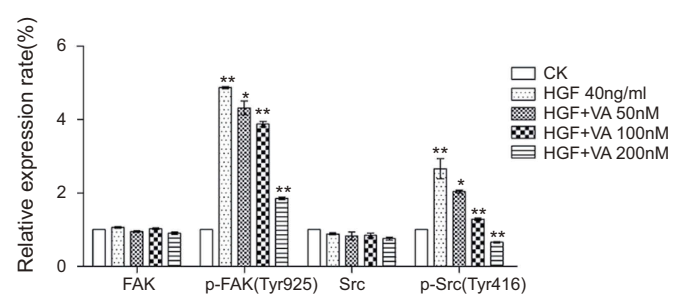

Figure 3 Verticillin A inhibits HGF-induced activation of c-Met/FAK/Src pathway. AGS cells were pretreated with increasing doses of verticillin A (0, 50, I00, tumor200 $\mathrm{nM}$ ) for $24 \mathrm{hrs}$ prior to HGF stimulation $(40 \mathrm{ng} / \mathrm{mL})$ for 5 mins. HeLa cells were pretreated with varying concentrations of verticillin $A(0,100,200,400 \mathrm{nM})$ for 24 hrs prior to HGF stimulation $(40 \mathrm{ng} / \mathrm{mL})$ for 20 mins. Cells were then harvested and analyzed by Western blotting analysis for p-Met(Tyrl234/35), FAK, p-FAK(Tyr925), Src, p-Src (Tyr4I6). The relative protein levels as shown in ( $\mathbf{A}$ and $\mathbf{C})$ were quantified and presented in ( $\mathbf{B}$ and $\mathbf{D})$, respectively. The data shown in (B and $D)$ are the mean \pm SD from three independent experiments. $* P<0.05$ and $* * P<0.01$. VA represented Verticillin $A$ in this figure.

\section{Overexpression of FAK increases human} gastric and cervical cancer cells migration

To further determine the function of verticillin A in the suppression of tumor progression through inhibiting the FAK/Src signaling pathway, we transiently transfected AGS and HeLa cells with either a FAK-expressing construct or an empty vector. FAK level increased about eightfold in AGS and HeLa cells 24 hrs after transfection with FAK-expressing 


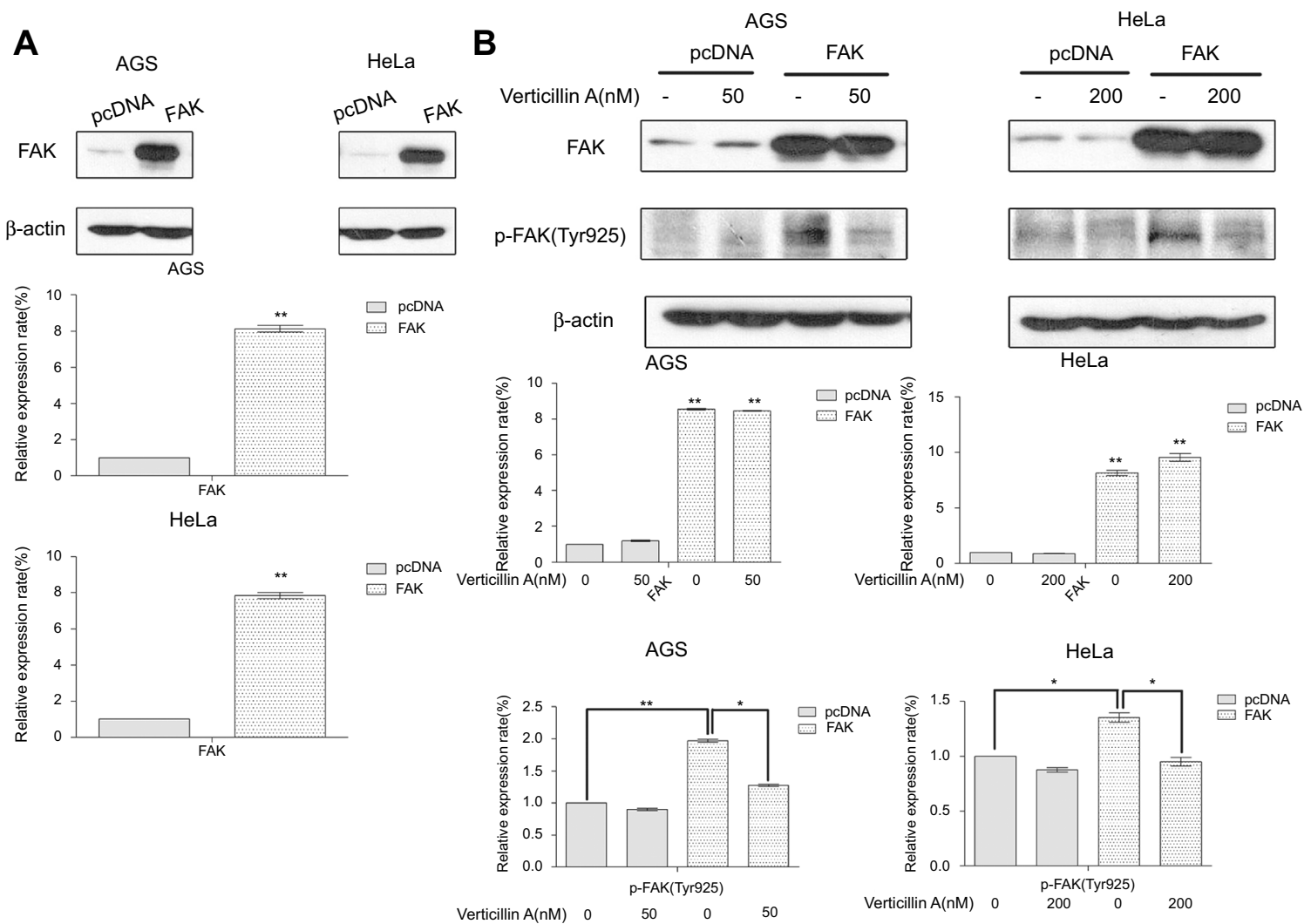

C

AGS

$0 \mathrm{nM}$

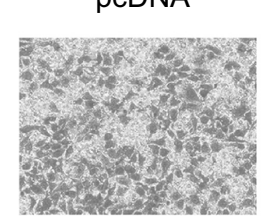

$50 \mathrm{nM}$

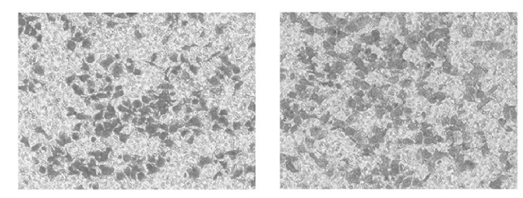

FAK

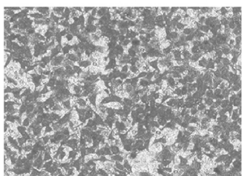

AGS

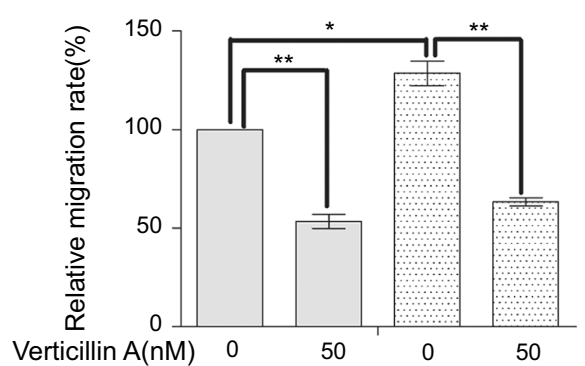

HeLa

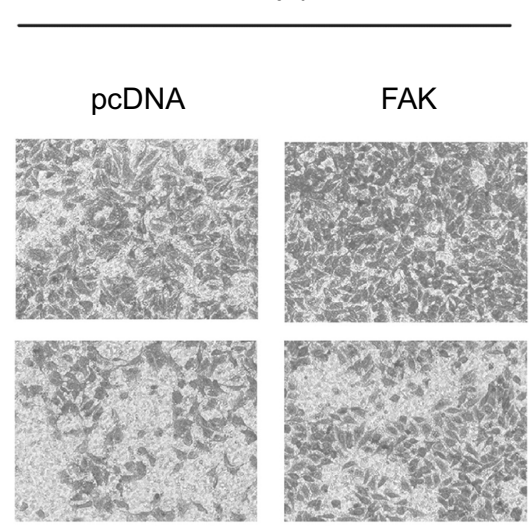

HeLa

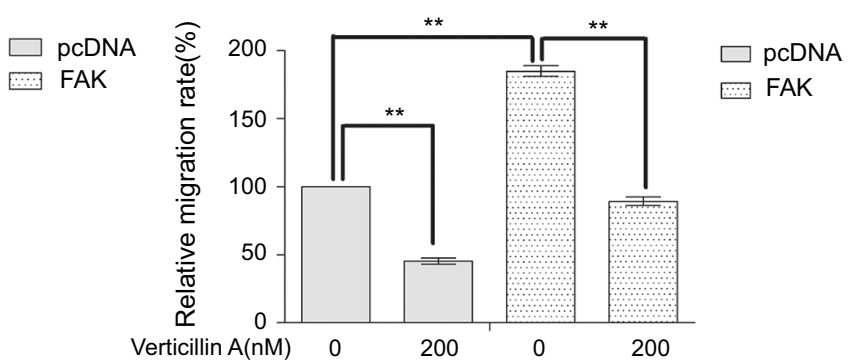

Figure 4 Verticillin A inhibits FAK overexpression-induced tumor cell migration. (A) AGS and HeLa cells were transiently transfected with a FAK-expressing construct or an empty vector for $24 \mathrm{hrs}$. Cells were analyzed by Western for the expression level of FAK. The protein levels were quantified and presented at the bottom panels. *P<0.05 and **P<0.01. (B) The transfected tumor cells were treated with verticillin A at 50nM (for AGS cells) and $200 \mathrm{nM}$ (for HeLa cells) for 24 hrs, and analyzed by Western blotting analysis for FAK protein level. The protein levels were quantified and presented at the bottom panels. $* P<0.05$ and $* * P<0.01$. (C) The transfected tumor cells were seeded onto the upper chamber and treated with verticillin A for 24 hrs. Cell migratory abilities were measured by migration chamber assay. The relative quantitative determinations of migrated cells were calculated with 5 fields counted per experiment. The data shown here are the mean \pm SD from three independent experiments. $*<0.05$ and $* * P<0.01$. 
vector as compared to the empty vector-transfected cells (Figure 4A). Interestingly, p-FAK (Tyr925) level was also increased (Figure 4B). Consistent with the increased p-FAK protein level, both FAK-overexpressing AGS and HeLa cells showed significant increases in their migration abilities as compared to the control cells (Figure 4C). These observations indicate that overexpression and activation of FAK increase gastric and cervical cancer cells migration capability.

We next examined the efficacy of verticillin A in inhibition of FAK activation and cancer cell migration. Verticillin A treatment significantly decreased p-FAK protein level without change total FAK protein level (Figure 4B). Consistent with inhibition of $p$-FAK, verticillin A treatment significantly decreased the migration capability of FAK-overexpressing in both gastric and cervical cancer cells (Figure 4C).

\section{Verticillin A decreases c-Met expression through inhibition of fatty acid synthase}

To further elucidate the mechanism underlying verticillin A-mediated decrease of c-Met protein level, we analyzed AGS and HeLa cells treated with various concentrations of verticillin A. As shown in Figure 5A, verticillin A treatment decreased the total c-Met protein level in a dose-dependent manner. We then treated AGS and HeLa cells with the fatty acid synthase (FASN) inhibitor C75. It is clear that C75 treatment also decreased c-Met protein level in a dosedependent manner (Figure 5B), suggesting that there is a link between FASN activity and c-Met expression. To delineate the relationship between verticillin A and FASN in c-Met expression, gastric and cervical cancer cells were treated with palmitate, the end product of FASN catalytic activity, and analyzed for c-Met protein level. Palmitate prevented verticillin A-induced decrease of c-Met in a dose-dependent manner in both gastric and cervical cancer cells (Figure 5C). Taken together, our observations indicate that verticillin A represses FASN to decrease c-Met protein level in gastric and cervical cancer cells.

\section{Discussion}

Extensive evidence suggest that the HGF/c-Met signaling pathway is aberrantly activated in various types of human cancers and promote cancer cells growth and metastasis. ${ }^{25}$ Expression of c-Met was found overexpressed, activated, and sometimes mutated inNSCLC tumor tissues, advanced cervical tumor tissues and gastric tumor tissues. c-Met overexpression could be a negative prognostic factor of these carcinoma. ${ }^{26-30}$ Recently, therapeutic approaches targeting the HGF/c-Met signaling pathway have shown great promises in cancer treatment in a number of clinical trials, including, lung, kidney, pancreatic and cervical cancers. ${ }^{11,12,14,31}$ In this study, we determined that verticillin A is effective in suppressing gastric and cervical cancer cells migration and invasion in vitro. Verticillin A was originally identified in Verticillium, an imperfect fungus isolated from a basidiocarp of Coltricia cinnamomea (Polystictus cinnamomeus), and identified as a small molecule member of a large and diverse family of epipolythiodioxopiperazine alkaloids released in response to pathogen infection. ${ }^{32}$ We have previously shown that verticillin A is effective in suppression of metastatic human colorectal cancer cell growth and in induction of tumor cell apoptosis. ${ }^{17,33}$ More importantly, verticillin A is a potent apoptosis sensitizer in human colon carcinoma cells which were resistant to TRAIL- or Fas- and other cytotoxic agent-induced apoptosis in vitro at nanomolar concentrations. ${ }^{17,18}$ These previous studies determined that verticillin A is effective in suppressing tumor growth. Our findings in this study extend verticillin A function as an anti-cancer metastasis agent that effectively suppresses tumor cell migration and invasion.

At the molecular level, we revealed that c-Met is a molecular target of verticillin A. Verticillin A is effective in inhibition of c-Met phosphorylation induced by HGF. Furthermore, we determined that verticillin A also reduces total c-Met protein level through inhibition of FASN, revealing a previously uncharacterized link between FASN activity and cMet expression in human gastric and cervical cancer cells. However, the mechanism underlying FASN regulation of cMet protein is not clear. One possible explanation is that inhibition of FASN disrupts the lipid raft, a plasma membrane microdomain, to cause a decrease in c-Met protein. FASN is the sole enzyme responsible for synthesis of long-chain saturated fatty acids and may play a role in regulating the activity of lipid rafts. ${ }^{34}$ And. a connection between lipid rafts and c-Met level has been reported. ${ }^{35}$ FASN has been shown to reprogram the epigenome in uterine leiomyosarcomas to promote H3K9me3 deposition in tumor cells. ${ }^{36}$ It is therefore also possible that FASN may induce H3K9me3 deposition to regulate c-Met expression in cancer cells, which remains to be determined.

The c-Met downstream signaling modulators are common to many RTK. Intracellularly, c-Met contains the catalytic tyrosines Y1234 and Y1235, which positively modulate the enzyme activity, while the juxtamembrane tyrosine 1003 negatively regulates c-Met by recruiting the ubiquitin ligase casitas B-lineage lymphoma (c-CBL). Tyrosine residues Y1349 and Y1356 in the C-terminal tail of c-Met act as multifunctional 
A
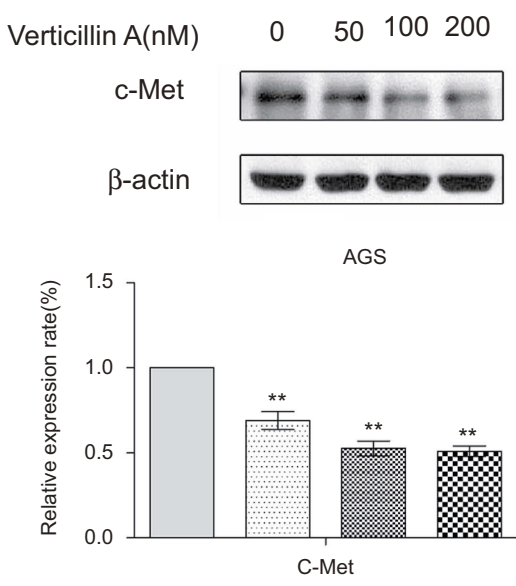

B

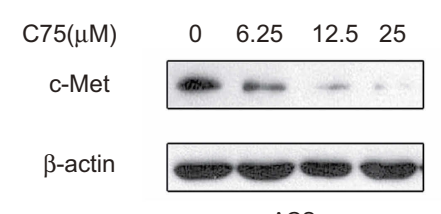

AGS

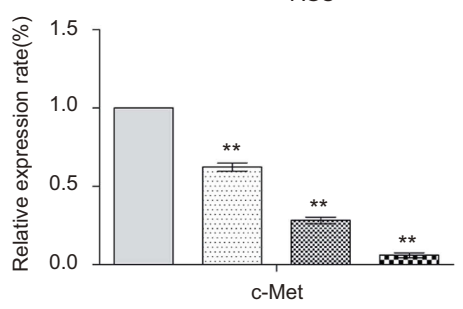

C
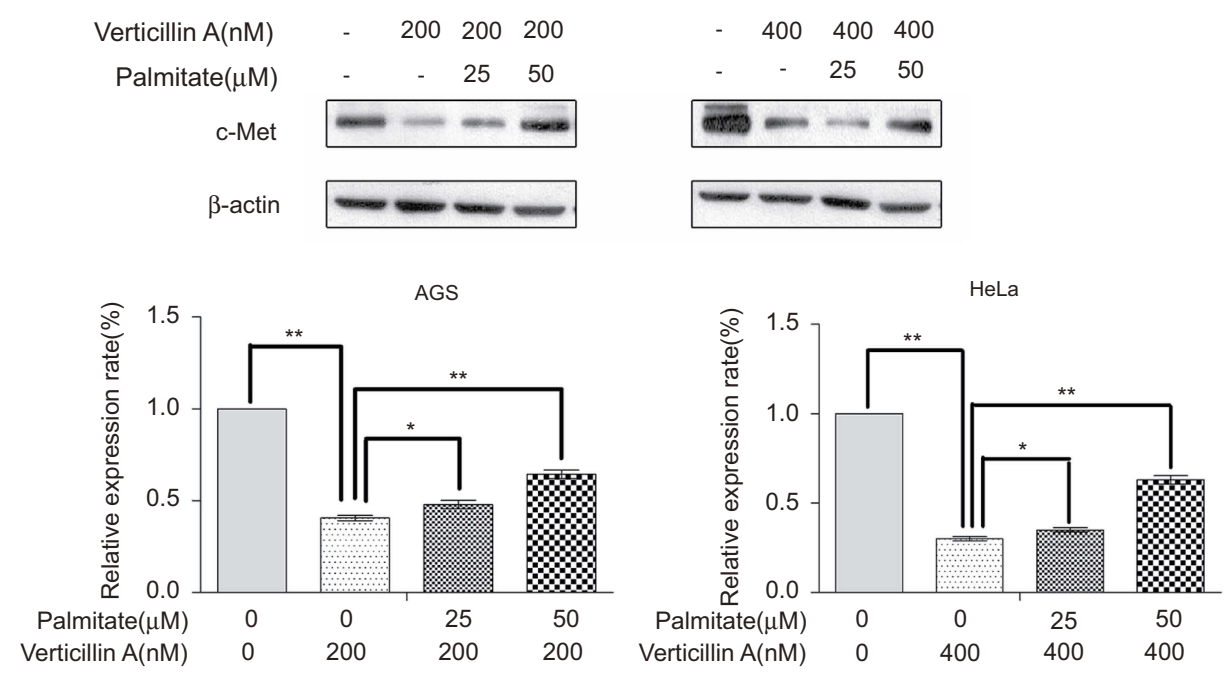

HeLa

\section{$\begin{array}{llll}0 & 100 & 200 & 400\end{array}$}
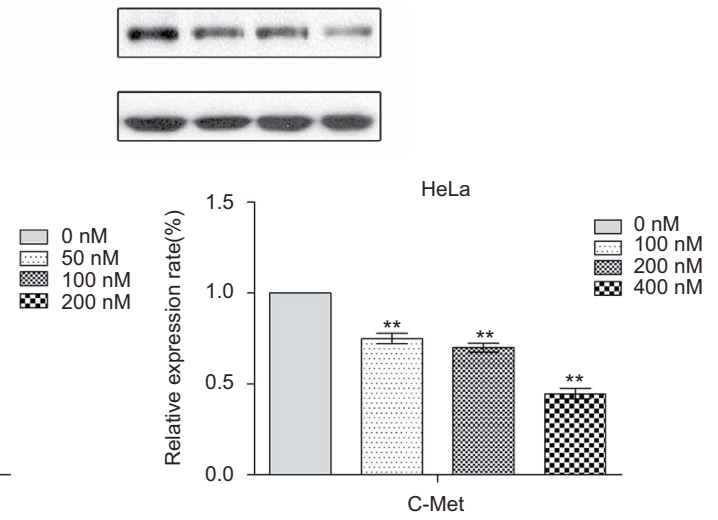

HeLa
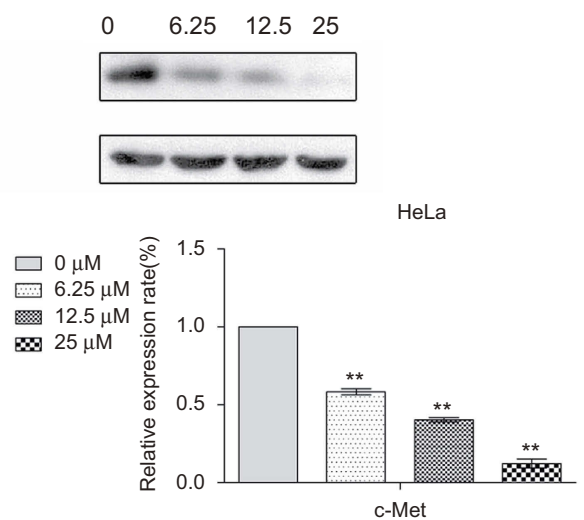

HeLa $\square 0 \mu \mathrm{M}$

$6.25 \mu \mathrm{M}$

$12.5 \mu \mathrm{M}$

\$8 $25 \mu \mathrm{M}$

Figure 5 Verticillin A reduces c-Met level through inhibition of fatty acid synthase (FASN). (A) AGS and HeLa cells were treated with varying concentrations of verticillin A for $24 \mathrm{hrs}$, and then analyzed by Western blotting for c-Met protein level. The levels of c-Met protein as shown in the top panel were quantified and presented at the bottom panel. $* P<0.05$ and $* * P<0.01$. (B) AGS and HeLa cells were treated with $C 75$ at the indicated concentrations for 24 hrs and then analyzed by Western blotting for $c-M e t$ protein. The levels of c-Met protein as shown in the top panel were quantified and presented at the bottom panel. $* P<0.05$ and $* * P<0.0$ I. (C) AGS and HeLa cells were treated with verticillin $A$ at the indicated concentrations for 24 hrs in the absence or presence of palmitate at the indicated concentrations. Cells were then analyzed by Western blotting for c-Met protein level. The levels of c-Met protein as shown in the top panel were quantified and presented at the bottom panel. $* P<0.05$ and $* * P<0.01$. The data shown here are the mean \pm SD from three independent experiments. 
docking sites for intracellular adaptor proteins when they were phosphorylated. ${ }^{9}$ HGF-induced c-Met receptor homodimerization activates the receptor tyrosine kinase by phosphorylation of tyrosine residues 1234 and 1235. Consequent phosphorylation and recruitment activate various downstream signaling cascades, including ERK/MAPK, PI3K/Akt, PIP3PKC, STAT3-JNK and FAK ${ }^{37}$ We determined that FAK is one of the downstream signaling modulators that were affected by verticillin A. FAK protein, a well-known cytoplasmic tyrosine activated on cell adhesion, mediates cell adhesion signaling in regulation of cell migration, survival, and differentiation, is often overexpressed and activated in various cancers. ${ }^{38}$ FAK can be autophosphorylated at tyrosine Y397, and Src binds to Y397 site, this FAK/Src complex further phosphorylates the activation loop Y576/577 site. Several additional trans-phosphorylation sites, Y861 and Y925 provides binding sites for SH2 (Src homology-2) domain-containing proteins. These sites ultimately regulate FAK kinase activity. ${ }^{10,38}$ Our study demonstrated that overexpression of FAK in gastric and cervical cancer cells resulted in a significant increase in these cells migratory ability. HGF-induced c-Met phosphorylation further activated FAK at tyrosine 925, which can be inhibited by verticillin A.

In conclusion, we determined that verticillin $\mathrm{A}$ is a potent suppressor of tumor migration and invasion. Verticillin A suppresses gastric and cervical cancer cells migration and invasion through inhibiting the c-Met/FAK/Src signaling pathway. Our observation suggested that verticillin A is potentially an effective agent for suppression of human gastric and cervical cancers metastasis.

\section{Acknowledgments}

This study is supported by National Natural Science Foundation of China (No. 31570811), Natural Science Foundation of Zhejiang Province (LY15C020001) and SiYuan Foundation.

\section{Disclosure}

The authors report no conflicts of interest in this work.

\section{References}

1. Terry MB, Gaudet MM, Gammon MD. The epidemiology of gastric cancer. Semin Radiat Oncol. 2002;12:111-127.

2. Torre LA, Bray F, Siegel RL, Ferlay J, Lortettieulent J, Jemal A. Global cancer statistics, 2012. Ca A Cancer J Clin. 2015;65:87-108. doi:10.3322/caac. 21294
3. Wui-Jin K, Greer BE, Abu-Rustum NR, et al. Cervical cancer, version 2.2015. J Natl Compr Canc Netw JNCCN. 2015;13:395-404. doi:10.6004/jncen.2015.0055

4. Zugazagoitia J, Guedes C, Ponce S, Ferrer I, Molina-Pinelo S, Pazares L. Current challenges in cancer treatment. Clin Ther. 2016;38:1551-1566. doi:10.1016/j.clinthera.2016.03.026

5. Steeg PS. Targeting metastasis. Nat Rev Cancer. 2016;16:201-218. doi:10.1038/nrc.2016.25

6. Lambert AW, Pattabiraman DR, Weinberg RA. Emerging biological principles of metastasis. Cell. 2017;168:670-691. doi:10.1016/j. cell.2016.11.037

7. Chaffer CL, Weinberg RA. A perspective on cancer cell metastasis. Science. 2011;331:1559-1564. doi:10.1126/science.1203543

8. David TW, Jennifer DH, Ermanno G, George VW, Eric XH. Structural basis for agonism and antagonism of hepatocyte growth factor. Proc Natl Acad Sci U S A. 2010;107:13264-13269. doi:10.1073/pnas.0910097107

9. Shawna Leslie O, Ming-Sound T. An overview of the c-MET signaling pathway. Ther Adv Med Oncol. 2011;3:S7-S19. doi:10.1177/ 1758834011422556

10. Lee BY, Timpson P, Horvath LG, Daly RJ. FAK signaling in human cancer as a target for therapeutics. Pharmacol Ther. 2015;146:132149. doi:10.1016/j.pharmthera.2014.10.001

11. Sharareh G, Hamid F, Mina M, Reza M, Fatemeh Homaei S, Amir A. Targeting c-MET/HGF signaling pathway in upper gastrointestinal cancers: rationale and progress. Curr Drug Targets. 2014;15:1302-1311. doi:10.2174/1389450115666141107105456

12. Boromand N, Hasanzadeh M, Sales SS, et al. Clinical and prognostic value of the C-Met/HGF signaling pathway in cervical cancer: c-Met/ HGF pathway in cervical cancer. J Cell Physiol. 2017;233:44904496. doi: $10.1002 /$ jcp.v233.6

13. Barrow-Mcgee R, Kermorgant S. Met endosomal signalling: in the right place, at the right time. Int J Biochem Cell Biol. 2014;49:69-74. doi:10.1016/j.biocel.2014.01.009

14. Garajová I, Giovannetti E, Biasco G, Peters GJ. c-Met as a target for personalized therapy. Transl Oncogenomics. 2015;7:13-31.

15. Demkova L, Kucerova L. Role of the HGF/c-MET tyrosine kinase inhibitors in metastasic melanoma. Mol Cancer. 2018;17:26. doi:10.1186/s12943-018-0795-z

16. Comoglio PM, Silvia G, Livio T. Drug development of MET inhibitors: targeting oncogene addiction and expedience. Nat Rev Drug Discov. 2008;7:504-516. doi:10.1038/nrd2530

17. Feiyan L, Qianqian L, Dafeng Y, et al. Verticillin A overcomes apoptosis resistance in human colon carcinoma through DNA methylation-dependent upregulation of BNIP3. Cancer Res. 2011;71:68076816. doi:10.1158/0008-5472.CAN-11-1575

18. Paschall AV, Dafeng Y, Chunwan L, et al. H3K9 trimethylation silences fas expression to confer colon carcinoma immune escape and 5-fluorouracil chemoresistance. J Immunol. 2015;195:18681882. doi:10.4049/jimmunol.1402243

19. Lu C, Yang D, Sabbatini ME, et al. Contrasting roles of H3K4me3 and $\mathrm{H} 3 \mathrm{~K} 9 \mathrm{me} 3$ in regulation of apoptosis and gemcitabine resistance in human pancreatic cancer cells. BMC Cancer. 2018;18:149. doi: $10.1186 / \mathrm{s} 12885-018-4242-8$

20. Fulda S. Tumor resistance to apoptosis. Int J Cancer. 2010;124:511515. doi:10.1002/ijc. 24064

21. Yokoyama Y, Hieda M, Nishioka Y, et al. Cancer-associated upregulation of histone $\mathrm{H} 3$ lysine 9 trimethylation promotes cell motility in vitro and drives tumor formation in vivo. Cancer Sci. 2013;104:889895. doi: $10.1111 /$ cas. 12166

22. Jahangiri A, Nguyen A, Chandra A, et al. Cross-activating c-Met $/ \beta 1$ integrin complex drives metastasis and invasive resistance in cancer. Proc Natl Acad Sci U S A. 2017;114:E8685-E8694. doi:10.1073/ pnas. 1701821114 
23. Mitra SK, Schlaepfer DD. Integrin-regulated FAK-Src signaling in normal and cancer cells. Curr Opin Cell Biol. 2006;18:516-523. doi:10.1016/j. ceb.2006.08.011

24. Hui AY, Meens JA, Colleen S, et al. Src and FAK mediate cell-matrix adhesion-dependent activation of met during transformation of breast epithelial cells. J Cell Biochem. 2010;107:1168-1181. doi:10.1002/ jcb.22219

25. Matsumoto K, Umitsu M, Silva DMD, Roy A, Bottaro DP. Hepatocyte growth factor/MET in cancer progression and biomarker discovery. Cancer Sci. 2017;108(3):296-307. doi:10.1111/cas.13156

26. Ma PC, Jagadeeswaran R, Jagadeesh S. Functional expression and mutations of c-Met and its therapeutic inhibition with SU11274 and small interfering RNA in non-small cell lung cancer. Cancer Res. 2005;65(4):1479-1488. doi:10.1158/0008-5472.CAN-04-4557

27. Cappuzzo F, Marchetti A, Skokan M, et al. Increased MET gene copy number negatively affects survival of surgically resected non-smallcell lung cancer patients. J Clin Oncol Off J Am Soc Clin Oncol. 2009;27:1667-1674. doi:10.1200/JCO.2008.19.1635

28. Baykal C, Ayhan AY, Al A, Yüce K, Ayhan A. Overexpression of the cMet/HGF receptor and its prognostic significance in uterine cervix carcinomas. Gynecol Oncol. 2003;88:123-129. doi:10.1016/S00908258(02)00073-2

29. Refaat T, Donnelly ED, Sachdev S, et al. c-Met overexpression in cervical cancer, a prognostic factor and a potential molecular therapeutic target. Am J Clin Oncol. 2015;93:E252-E252.

30. Kawakami H, Okamoto I. MET-targeted therapy for gastric cancer: the importance of a biomarker-based strategy. Gastric Cancer 2016;19:687-695. doi:10.1007/s10120-015-0585-x
31. Cuneo KC, Morgan MA, Griffith KA, et al. Prognostic value of c-MET expression in patients with pancreatic cancer receiving adjuvant and neoadjuvant chemoradiation therapy. Int $J$ Radiat Oncol Biol Phys. 2018;100:490-497. doi:10.1016/j.ijrobp.2 017.10 .030

32. Katagiri K, Sato K, Hayakawa S, Matsushima T, Minato $H$, Verticillin A. a new antibiotic from verticillium sp. $J$ Antibiot. 1970;23:420-422. doi:10.7164/antibiotics.23.420

33. Zewdu A, Lopez G, Braggio D, et al. Verticillin A inhibits leiomyosarcoma and malignant peripheral nerve sheath tumor growth via induction of apoptosis. Clin Exp Pharmacol. 2016;6:221.

34. Uddin S, Hussain AR, Ahmed M, et al. Inhibition of fatty acid synthase suppresses c-Met receptor kinase and induces apoptosis in diffuse large B-cell lymphoma. Mol Cancer Ther. 2010;9:1244-1255. doi:10.1158/1535-7163.MCT-10-0338

35. Zeng J, Zhang H, Tan Y, et al. Aggregation of lipid rafts activates cmet and c-Src in non-small cell lung cancer cells. BMC Cancer. 2018;18:611. doi:10.1186/s12885-018-4242-8

36. Guan M, Wu X, Chu P, Chow WA. Fatty acid synthase reprograms the epigenome in uterine leiomyosarcomas. PLoS One. 2017;12: e0179692. doi:10.1371/journal.pone.0179692

37. Carmen B, Walter B, Ermanno G, Woude GFV. Met, metastasis, motility and more. Nat Rev Mol Cell Bio. 2003;4:915-925. doi:10.1038/nrm1261

38. Sulzmaier FJ, Christine J, Schlaepfer DD. FAK in cancer: mechanistic findings and clinical applications. Nat Rev Cancer. 2014;14:598610. doi:10.1038/nrc3792
OncoTargets and Therapy

\section{Publish your work in this journal}

OncoTargets and Therapy is an international, peer-reviewed, open access journal focusing on the pathological basis of all cancers, potential targets for therapy and treatment protocols employed to improve the management of cancer patients. The journal also focuses on the impact of management programs and new therapeutic agents and protocols on patient perspectives such as quality of life, adherence and satisfaction. The manuscript management system is completely online and includes a very quick and fair peer-review system, which is all easy to use. Visit http://www.dovepress.com/ testimonials.php to read real quotes from published authors. 